\title{
INICIACIÓN DEL DESARROLLO PROFESIONAL DE LOS DOCENTES DE EDUCACIÓN PRIMARIA (ARGENTINA, COLOMBIA, ESPAÑA Y FRANCIA). ESTUDIO COMPARADO
}

\author{
Initiation of the professional development of the teachers of primary (Argentine, \\ Colombia, Spain and France). Comparative study.
}

\author{
Joan María Senent Sánchez \\ Miriam Llorente Rodríguez \\ Fecha de recepción: 20/10/2016 \\ Fecha de aceptación: 13/11/2016
}

RESUMEN: Uno de los aspectos fundamentales en la consecución de una educación de calidad es la figura del maestro, su formación y su forma de intervenir en las aulas de educación primaria en tanto en cuanto la profesionalidad de los docentes puede marcar la diferencia en el aprendizaje de los estudiantes incluso contrarrestando las dificultades de origen. De hecho: "Las escuelas que desafían sus condiciones adversas y logran buenos resultados de aprendizaje son aquellas en las que los docentes han asumido una mayor participación en la toma de decisiones institucionales y un mayor compromiso con los resultados de aprendizaje de los alumnos (OREALC/ UNESCO, 2007, p.60)". Por todo ello, desde este planteamiento, pretendemos analizar comparativamente los sistemas de formación e iniciación al desarrollo profesional (en su fase de inducción) de los docentes de primaria de Argentina y Colombia -con respecto a Latinoamérica- y España y Francia -con respecto a Europa. Seleccionamos esta muestra de países en la medida que consideramos que cumplen criterios de similitud mínimos, así como suficientes diferencias como para enriquecer la comparación. Para ello partiendo de variables como modelo formativo, formación previa a la iniciación docente, estructura de la formación e iniciación de su desarrollo profesional, realizamos una comparación cuyos resultados apuntan a la relación entre los modelos de formación y de acceso al trabajo y la repercusión cualitativa de la intervención en las aulas.

PALABRAS CLAVE: formación inicial docente, desarrollo profesional docente, educación primaria, educación comparada e internacional.

ABSTRACT: One of the key aspects in achieving quality education is the figure of the teacher, their training and how to intervene in elementary classrooms as long as the professionalism of teachers can make a difference in student learning even counteracting the difficulties of origin. In fact, schools that challenge their adverse conditions and achieve good learning results are those in which teachers have assumed greater participation in institutional decision-making and commitment to the results of student learning (OREALC / UNESCO, 2007, p. 60). Therefore, from this approach, we intend to analyze comparatively training systems and initiation to professional development (in their induction phase) of the primary teachers of Argentina and Colombia, with regard to Latin America- and Spain and France, with respect to Europe. We selected this sample of countries as we consider that meet minimum criteria of similarity and enough differences to enrich comparison. To do this based on variables such as training model, prior to teaching initiation formation, structure formation 
and initiation of their professional development; we make a comparison whose results point to the relationship between training models and access to work and qualitative impact intervention in the classroom.

KEYWORDS: initial teacher training, teacher professional development, primary education, comparative and international education.

\section{Introducción}

El presente trabajo parte de la convicción de que la educación es un elemento trascendental e insustituible para el desenvolvimiento integral del ser humano. Pero no cualquier tipo de formación es válida a la hora de cumplir con este noble objetivo. Por ello, es necesario cuidar de los diversos elementos que posibilitan que ésta sea de calidad. En este sentido, la convicción de que la figura del maestro es fundamental en la consecución de una educación de calidad es un hecho que parece consolidarse a la luz de un vasto corpus teórico de conocimiento.

Así pues, el estudio riguroso y en profundidad de la preparación inicial que los futuros maestros reciben antes de afrontar el proceso de enseñanza-aprendizaje con los que serán sus alumnos a lo largo de su carrera profesional, así como el proceso de iniciación al desarrollo profesional, se estiman imprescindibles para ofrecer las claves, las valoraciones y las conclusiones pertinentes que permitan vincular, en gran medida,- si bien no completamente, pues el concepto de calidad va mucho más allá-, dichos procesos de formación e iniciación profesional, en nuestro caso del profesorado de educación primaria, con la garantía y mejora de los resultados de aprendizaje y, en general, de la educación de los adultos del mañana.

Para tratar de ofrecer una mayor generalización de los resultados no nos circunscribiremos exclusivamente al ámbito nacional, sino que ampliaremos bajo una mirada internacional la perspectiva de análisis, de modo que nos permita comparar diferentes modelos de formación docente inicial que sean suficientemente representativos de la diversidad formativa existente en este ámbito, enriqueciendo así dicha comparación, al tiempo que comparten ciertos criterios de similitud, permitiendo así una correcta comparabilidad de los mismos. De este modo, una vez hecha esta justificación, diremos que los países seleccionados han sido Argentina y Colombia -con respecto a Latinoamérica- y España y Francia -con respecto a Europa.

El interés de los autores por el desarrollo profesional docente, del cual se deriva este estudio, está adscrito a un proyecto competitivo de amplia envergadura concebido en el seno del Grupo de Investigación en Políticas Educativas Supranacionales (GIPES) de la Universidad Autónoma de Madrid, bajo el título: "La iniciación en el desarrollo profesional docente en la educación obligatoria: de las políticas supranacionales a las trayectorias profesionales" (EDU2015-65743-P).

Concretamente, se enmarca en el objetivo segundo del proyecto el cual pretende analizar las políticas educativas contemporáneas acerca de la iniciación en el desarrollo profesional docente y los modelos e ideas que se promueven al respecto. En este sentido, de entre las diferentes metodologías de las cuales parte este objetivo para cumplir con los tres sub-objetivos que lo componen, nosotros nos hemos decantado por el estudio internacional basándonos en el análisis comparado. 


\section{Método}

La metodología que va a emplearse en este trabajo consiste en la utilización del método comparado en educación, no sin antes reparar en el hecho de que existe una pluralidad de métodos que son ampliamente aceptados por la mayoría de comparatistas como susceptibles de ser empleados en Educación Comparada, apartándonos de la creencia en la existencia de un único método correcto (García Garrido, García Ruiz, 2012). En este mismo sentido se manifiesta J. Caïs (2002). En la actualidad se vienen haciendo numerosos avances en el campo teórico de la Educación Comparada e Internacional que matizan, amplían y, en definitiva, desbordan las etapas comparativas del método clásico propuesto por Bereday (1964) y Hilker (1968) que son: descripción, interpretación, yuxtaposición y comparación. No obstante, el reconocimiento de estas cuatro etapas básicas en el método comparado goza de cierta aceptación entre los comparatistas de la educación.

Teniendo en consideración las palabras de Scriewer (1993) al señalar que no sólo se ha de realizar un análisis de los cambios teóricos de la Educación Comparada, sino también de las consecuencias metodológicas de dichos cambios, compartimos las valiosas matizaciones y aportaciones realizadas por García Garrido (1982) y Ferrer Julià (1990) al método de Bereday y Hilker que puede considerarse "el método comparativo por excelencia y con un mayor peso en la historia de la educación comparada contemporánea" (Ferrer Julià, 1990, p.75).

Una vez hechas estas aclaraciones, siguiendo la clasificación de Roselló (1978) diremos que nuestra investigación comparativa tiene una naturaleza explicativa pues más allá del establecimiento de semejanzas y diferencias pretende encontrar las causas de las mismas. Se realiza en un momento temporal determinado, en la actualidad, entre cuatro unidades de comparación, por lo que se trata de una comparación estática o sincrónica. En función de las unidades de comparación, nuestro estudio atiende los países latinoamericanos de Argentina y Colombia y a los europeos de Francia y España (unidades de comparación).

En la fase de yuxtaposición las unidades de comparación serán contrastadas unas con otras mediante cuadros o tablas en torno a unos criterios de comparación que podemos concretar de la siguiente forma:

- Estructura del sistema educativo

- Modelo de formación inicial

- Requisitos específicos de acceso a la formación inicial docente

- \% de estudiantes proveniente de Formación Profesional

- Nivel de la formación inicial docente (CINE)

- Organización de la formación inicial docente

- Iniciación al desarrollo profesional docente

Posteriormente hemos realizado una interpretación de la yuxtaposición comparativa haciendo referencia a cada uno de los criterios, lo que nos ha permitido llegar a una serie de reflexiones que se especifican en el apartado de conclusiones. 


\section{Resultados y análisis}

\subsection{Formación inicial e iniciación profesional del docente de educación primaria en España, Argentina, Francia y Colombia.}

Sin más dilación, una vez hechos los anteriores posicionamientos, pasamos a realizar el análisis comparado anunciado en lo que concierne a la formación inicial docente y a la iniciación profesional de los mismos en los países de España, Argentina, Colombia y Francia.

Tabla 1: Comparación de la formación inicial e iniciación profesional del docente de educación primaria (España, Argentina, Francia y Colombia).

\begin{tabular}{|c|c|c|c|c|c|}
\hline \multicolumn{2}{|l|}{$\begin{array}{l}\text { País } \\
\text { Variable }\end{array}$} & España & Argentina & Francia & Colombia \\
\hline \multicolumn{2}{|c|}{$\begin{array}{l}\text { Estructura del sistema } \\
\text { educativo }^{1}\end{array}$} & Comprensivo & Comprensivo & $\begin{array}{l}\text { Semi-comprensivo } \\
\text { (comprensivo hasta } \\
\text { el final del Collège } \\
\text { (15 años) }\end{array}$ & $\begin{array}{l}\text { Comprensivo } \\
\text { Educación básica 4- } \\
14 \text { años) = } \\
\text { obligatoriedad } \\
\text { escolar }\end{array}$ \\
\hline \multicolumn{2}{|c|}{ Modelo de formación inicial $^{2}$} & Simultáneo & Simultáneo & Consecutivo & Simultáneo \\
\hline \multicolumn{2}{|c|}{$\begin{array}{l}\text { Requisitos específicos acceso a } \\
\text { la formación inicial docente }\end{array}$} & No & Sí & $\begin{array}{l}\text { SI (Licence para } \\
\text { entrar en los } \\
\text { Masters de la } \\
\text { ESPE) }\end{array}$ & SI. Bachillerato \\
\hline \multicolumn{2}{|c|}{$\begin{array}{l}\text { \% de estudiantes proveniente } \\
\text { de Formación Profesional }\end{array}$} & $50-70 \%$ & 0 & $5 \%$ & 0 \\
\hline \multicolumn{2}{|c|}{$\begin{array}{l}\text { Nivel de la formación inicial } \\
\text { docente (CINE) }\end{array}$} & $\begin{array}{l}\text { Educación } \\
\text { superior } \\
\text { universitaria } \\
\text { (CINE 6) }\end{array}$ & $\begin{array}{l}\text { Educación } \\
\text { Superior no } \\
\text { universitaria }^{3} \\
\text { (CINE 5/6) }\end{array}$ & $\begin{array}{l}\text { Educación superior } \\
\text { universitaria. Nivel } \\
\text { Master. (CINE 7) }\end{array}$ & $\begin{array}{l}\text { Educación terciaria } \\
\text { (CINE 4/5) }\end{array}$ \\
\hline \multirow{4}{*}{$\begin{array}{l}\text { Organización de } \\
\text { la formación } \\
\text { inicial } \\
\text { Docente }\end{array}$} & $\begin{array}{l}\text { Años de } \\
\text { formación }\end{array}$ & 4 años & 4 años & $\begin{array}{l}2 \text { años específicos } \\
\text { con } 3 \text { años previos a } \\
\text { la entrada en la } \\
\text { ESPE. } 3 \\
\text { (licence)+1 (MEEF- } \\
\text { Master 1) + } \\
\text { concours + } 1 \\
\text { (MEES - Master } \\
\text { 2) } 1\end{array}$ & $\begin{array}{l}2,5 \text { años en las } \\
\text { Escuelas Normales } \\
\text { superiores. - } 2 \text { años } \\
\text { si vienen de un } \\
\text { bachiller } \\
\text { pedagógico) }\end{array}$ \\
\hline & $\begin{array}{l}\text { Edad } \\
\text { habitual en } \\
\text { final de } \\
\text { formación }\end{array}$ & 22 & 21 & $24-25$ & $17-18$ \\
\hline & $\begin{array}{l}\text { Formación- } \\
\text { Capacitació } \\
\text { n } \\
\text { profesional } \\
\text { específica }^{4}\end{array}$ & $\begin{array}{l}\text { Sí. } 87,5 \% \text { de la } \\
\text { carga lectiva. }\end{array}$ & $\begin{array}{l}\text { Sí. } 75 \% \text { de la } \\
\text { carga lectiva } 5\end{array}$ & $\begin{array}{l}70 \% \text { de los dos } \\
\text { años específicos }\end{array}$ & $\begin{array}{l}60 \% \text { de la carga } \\
\text { lectiva }\end{array}$ \\
\hline & $\begin{array}{l}\text { Período de } \\
\text { Prácticas }\end{array}$ & $\begin{array}{l}\text { SÍ Entre } 500 \text { y } \\
1100 \text { horas (De } 20 \\
\text { a } 44 \text { créditos } \\
\text { ECTS }^{6} \text { ). }\end{array}$ & $\begin{array}{l}\text { Sí } \\
\text { Entre } 390 \text { y } 650 \\
\text { horas?. }\end{array}$ & $\begin{array}{l}\text { SI } \\
300-400 \mathrm{~h} .\end{array}$ & $\begin{array}{l}\text { SI } \\
200 \mathrm{~h} .\end{array}$ \\
\hline
\end{tabular}




\begin{tabular}{|c|c|c|c|c|c|}
\hline \multicolumn{2}{|l|}{$\begin{array}{l}\text { País } \\
\text { Variable }\end{array}$} & España & Argentina & Francia & Colombia \\
\hline & $\begin{array}{l}\text { Trabajo } \\
\text { Final de } \\
\text { Investigaci } \\
\text { ón }\end{array}$ & $\begin{array}{l}\text { SÍ } \\
(\text { de } 6 \text { a } 30 \text { créditos } \\
\text { ECTS }^{8} \text { ) }\end{array}$ & NO & $\begin{array}{l}\text { SI } \\
8 \text { ECTS (de media) }\end{array}$ & $\mathrm{NO}$ \\
\hline \multirow{3}{*}{$\begin{array}{l}\text { Iniciación al } \\
\text { desarrollo } \\
\text { profesional } \\
\text { docente }\end{array}$} & $\begin{array}{l}\text { Medidas de } \\
\text { apoyo y/o } \\
\text { programas } \\
\text { de } \\
\text { inducción. }\end{array}$ & $\begin{array}{l}\text { Medidas de apoyo } \\
\text { (establecidas a } \\
\text { nivel de las } \\
\text { Comunidades } \\
\text { Autónomas) }\end{array}$ & $\begin{array}{l}\text { Medidas de apoyo } \\
\text { (establecidas a } \\
\text { nivel nacional }{ }^{9} \text { ) }\end{array}$ & $\begin{array}{l}\text { Medidas de apoyo } \\
\text { (establecidas a nivel } \\
\text { nacional) }\end{array}$ & $\begin{array}{l}\text { Medidas de apoyo } \\
\text { (establecidas a nivel } \\
\text { nacional) }\end{array}$ \\
\hline & $\begin{array}{l}\text { Método de } \\
\text { acceso al } \\
\text { trabajo }\end{array}$ & $\begin{array}{l}\text { Concurso- } \\
\text { oposición } \\
\text { Contrato en } \\
\text { enseñanza } \\
\text { concertada y } \\
\text { privada. }\end{array}$ & Concurso $^{10}$ & $\begin{array}{l}\text { Méritos (notas + } \\
\text { experiencias } \\
\text { docentes } \\
\text { anteriores). El } \\
\text { concurso se realiza } \\
\text { para acceder al } \\
\text { último año de } \\
\text { formación }\end{array}$ & Concurso \\
\hline & $\begin{array}{l}\% \text { de } \\
\text { titulados } \\
\text { trabajando } \\
3 \text { años } \\
\text { después de } \\
\text { acabar } \\
\text { estudios. }\end{array}$ & $20 \%$ & $\begin{array}{l}50 \% \text {. Muchos lo } \\
\text { comparten con } \\
\text { estudios } \\
\text { universitarios }\end{array}$ & $100 \%$ & $\begin{array}{l}40 \% \text {. Muchos } \\
\text { deciden continuar } \\
\text { estudios en la } \\
\text { universidad. }\end{array}$ \\
\hline
\end{tabular}

Fuentes: Eurydice, OEI-Red Quipu, Ministerios de Educación de los países seleccionados.

\section{NOTAS:}

${ }^{1}$ La estructura del sistema educativo de un país puede catalogarse como comprensivo, semicomprensivo o segregado. Llamamos sistema comprensivo aquel en el que el alumno/a de no debe pasar ningún filtro ni tomar ninguna opción para progresar de un curso a otro o de una a otra etapa. Durante los años de escolaridad obligatoria. Los sistemas semicomprensivos son básicamente sistemas comprensivos que en el último o en los dos últimos años de obligatoriedad escolar presentan alguna opción a realizar en función de la trayectoria escolar del alumno/a. Finalmente los sistemas segregados o diferenciados son aquellos en los que los alumnos/as deben pasar varios filtros o realizar varias opciones durante su etapa escolar obligatoria. Habitualmente estas opciones se realizan al finalizar cada etapa, en el paso de primaria a secundaria, y de secundaria inferior a la superior.

${ }^{2}$ Los modelos de formación inicial docente pueden ser simultáneos o consecutivos. Tal y como establece Comisión Europea/EACEA/Eurydice (2013) "la formación del profesorado puede organizarse de distintas formas, pero por lo general suele incluir un componente general y uno profesional. El componente general hace referencia a los cursos de formación general y al dominio de la(s) materia(s) que los candidatos van a enseñar una vez obtengan el título. La parte profesional proporciona a los futuros profesores las destrezas tanto teóricas como prácticas que van a necesitar para la enseñanza e incluye prácticas en el aula. Se pueden diferenciar dos grandes modelos de formación inicial del profesorado, en función de cómo se combinen estos dos componentes. Modelo simultáneo, cuando el componente profesional se cursa al mismo tiempo que el general o modelo consecutivo, cuando el componente profesional se cursa a continuación del general" (p.23). 
${ }^{3}$ La formación de profesores en el nivel universitario está generalizada en la educación media. No obstante, desde hace varios años diversas universidades nacionales ofrecen formación docente inicial para profesores de primaria (EGB1 y EGB2) (CFE, 2007a).

${ }^{4}$ Tal y como establece Comisión Europea/EACEA/Eurydice (2013) se entiende por capacitación profesional aquella que proporciona a los futuros profesores las competencias tanto teóricas como prácticas necesarias para la docencia. Incluye, además de cursos de psicología, teoría de la educación y metodología didáctica, prácticas en el aula.

${ }^{5}$ En el caso de Argentina, la formación se organiza en tres campos específicos (formación general, formación específica y formación en la práctica profesional). Para obtener un porcentaje comparable con el español y francés, en el caso de Argentina éste se ha obtenido estableciendo un promedio entre los porcentajes máximos y mínimos asignados a nivel nacional al campo de formación específica y de formación práctica.

${ }^{6}$ Elaboración propia en base a lo establecido en el Real Decreto 1393/2007, de 29 de octubre, por el que se establece la ordenación de las enseñanzas universitarias oficiales y la ORDEN ECI/3857/2007, de 27 de diciembre, por la que se establecen los requisitos para la verificación de los títulos universitarios oficiales que habiliten para el ejercicio de la profesión de Maestro en Educación Primaria y teniendo en consideración la equivalencia de 1 crédito $\mathrm{ECTS}=25$ horas.

${ }^{7}$ Cálculo establecido en función del peso relativo asignado al área de la formación práctica (entre un $15 \%$ y 20\%) (UNESCO/IBE, 2010) y el total de horas asignados a la formación inicial docente en primaria (2600 horas) (CFE, 2007b).

${ }^{8}$ Elaboración propia en base a lo establecido en el Real Decreto 1393/2007, de 29 de octubre, por el que se establece la ordenación de las enseñanzas universitarias oficiales.

${ }^{9}$ El Ministerio de Educación de la Nación venía desarrollando en varias provincias, el Proyecto de Acompañamiento a Docentes Noveles a través del Instituto Nacional de formación docente. No obstante, la línea de acción destinada al acompañamiento de maestros y profesores en sus primeros desempeños profesionales, como práctica regular y sistemática derivada de la acción estatal no es generalizada, sino que se desarrolla exclusivamente en los Institutos de Formación Superior seleccionados por las provincias.

${ }^{10}$ Según el artículo 61 del Estatuto docente (conforme sustitución por art. 1 de la Ley $\mathrm{N}^{\circ} 939$ ) se establece que el ingreso al ejercicio en la enseñanza primaria será por concurso. No obstante, se establece el complemento de pruebas de oposición en los casos en que se consideren necesarias. 


\subsection{Análisis de la yuxtaposición}

\subsubsection{Estructura del sistema educativo de las unidades de comparación}

En lo que concierne al tipo de estructura de los sistemas educativos, tanto España como Argentina y Colombia poseen sistemas comprensivos en los que la separación diferencial de los estudiantes en itinerarios diferenciados tiene lugar prácticamente al final de la escolaridad obligatoria. En estos países la elección tiene lugar en el tramo de edad de 14 a 15 años. En Colombia, concretamente, la escolaridad obligatoria y la comprensividad del sistema acaba a los 14 años. Si bien es cierto, en el caso de España, aunque hayas cursado un itinerario concreto, puedes presentarte a la evaluación final de la ESO por cualquiera de los dos itinerarios existentes, con independencia de la opción cursada, e incluso por ambos en la misma ocasión (MECD, 2013). En el caso francés, nos hallamos frente a un sistema fundamentalmente comprensivo, aun cuando al terminar el collège (15 años) el alumno/a debe elegir entre diferentes modalidades de Bachillerato.

\subsubsection{Modelo de formación inicial}

El modelo simultáneo de formación inicial del profesorado de educación primaria es aplicado en España, Colombia y en Argentina, ya que los estudiantes tienen la formación específica para el ejercicio de su futura actividad profesional desde que comienzan los estudios conducentes a la obtención del título. Por el contrario, el caso francés, tanto en la estructura anterior de los IUFM como en las actuales ESPE (Escuelas Superiores del Profesorado) es el único caso europeo donde aparece un planteamiento de la formación de carácter consecutivo, constituido por una licence (con más de 100 posibilidades diferentes) que da acceso a un Master 1, seguido del concurso - oposición y del Master 2, ambos de carácter pedagógico.

\subsubsection{Requisitos específicos de acceso a la formación inicial docente}

En España, como ocurre en gran parte de los países europeos, no existen requisitos de acceso específicos a la formación inicial docente. Éstos, más bien, están relacionados con el acceso, en general, a la educación superior (Comisión Europea/ EACEA/ Eurydice, 2013). Con la vigente ley orgánica para la mejora de la calidad de educativa (LOMCE) el acceso al nivel superior universitario vendrá establecido por la nota final del bachiller que se compone de una prueba individualizada (30\% de la nota final) y de la media obtenida del rendimiento académico de los dos cursos que componen el bachiller (60\%) al tiempo que las universidades podrán establecer, de forma excepcional, evaluaciones específicas de conocimientos y/o de competencias (MECD, 2013). A partir del curso 2017-2018 la prueba de acceso a la Universidad (PAU) que viene realizándose como único criterio de admisión a la universidad dará paso al sistema explicado.

Al mismo tiempo, también se permite el acceso a la formación inicial docente mediante determinadas especialidades afines del ámbito de la formación profesional superior. Numerosas universidades ofrecen pruebas de acceso a la misma para mayores de 25 para mayores de 45 años que también permiten a los aspirantes, una vez superada, ingresar en los estudios superiores universitarios, en nuestro caso, en los grados de Educación Infantil y Primaria. Finalmente, también podrán tener acceso a las enseñanzas de grado las personas mayores de cuarenta años con experiencia laboral o profesional en relación con una enseñanza. (MECD, 2014)

En Argentina, en la mayoría de los casos se exige un examen psicofísico que determine las condiciones de salud para el ejercicio de la docencia, independientemente de los relacionados con el acceso general a la educación superior. Respecto a los citados requisitos generales, en todos los casos se exige el título de nivel medio para acceder a estudios 
conducentes al desempeño docente. Por excepción, los mayores de 25 años que no reúnan esa condición podrán ingresar siempre que demuestren, a través de un examen, que tienen preparación y/o experiencia laboral docente acorde con los estudios que se proponen iniciar, así como aptitudes y conocimientos suficientes para cursarlos (OEI, 2003)

En Francia como antes se indicaba el acceso a la ESPE pasa por la obtención previa de una licence ( 3 años) con más de 100 opciones diferentes. Estos estudios en la mayoría de los casos no tienen relación con educación, mientras que si lo tienen los dos másteres que se realizan dentro de la ESPE.

Por último, en este sentido, la formación inicial de docentes, en Colombia, tiene tres rutas de profesionalización:

- La formación complementaria, ofrecida por las Escuelas Normales Superiores que conduce al título de normalista superior.

- La formación de pregrado de las Facultades de Educación, que otorga el título de licenciado(a), en determinada área del conocimiento (área curricular) con énfasis en un nivel de la escolaridad o en la atención a poblaciones específicas y permite el ejercicio de la docencia en los niveles y modalidades de acuerdo a la especialidad del programa.

- La ruta de formación establecida por el Decreto 1278 de 2002 que permite a los profesionales no licenciados optar por la profesión docente a través de la realización de un programa de pedagogía o el inicio de estudios de postgrado (especialización, maestría o doctorado) en educación.

\subsubsection{Organización de la formación inicial docente}

Los años de formación para obtener la titulación pertinente que habilite para el ejercicio de la docencia en el nivel primario de educación son diferentes en el conjunto de los países. En el contexto europeo los grados establecidos por el acuerdo de Bolonia implican 3 ó 4 años, aun cuando la formación se realiza a nivel de grado en España y a nivel de Máster en Francia. Argentina desarrolla una formación en 4 años especialmente implementada desde los Institutos Terciarios (CINE 5), mientras que, en el caso colombiano, la formación. Si bien es cierto, en el caso español, la formación corresponde al nivel de educación superior universitaria y en el caso de Argentina, eminentemente, al nivel de educación superior no universitaria.

En los dos países se llevan a cabo diversos períodos de prácticas, que en el caso de Argentina representan en torno a un $20 \%$ promedio de la carga lectiva de su programa formación inicial, mientras que en España este porcentaje es del 13,3\% promedio ${ }^{1}$. Pero sólo en España los estudiantes deben realizar un trabajo de investigación en el último curso de su formación para poder obtener el título (Trabajo Final de Grado).

\footnotetext{
$1 \quad$ Este promedio respeta el Real Decreto 1393/2007 que establece que los trabajos finales de grado tendrán una carga mínima de 6 créditos y máxima de 30 créditos. Siguiendo la ORDEN/ECI 3857/2007 el módulo de practicum, que tiene un peso específico de 50 créditos, contiene tanto practicas escolares como el Trabajo Final de Grado por lo que podemos establecer entre 44 y 20 créditos asignados al período de prácticas. Si bien es cierto, en la mayoría de titulaciones de grado en Educación Primaria de las universidades españolas, la carga lectiva asignada al Trabajo Final de Grado tan sólo oscila entre 6 y 10 créditos ECTS, lo que haría que el promedio calculado de prácticas fuera más elevado para los futuros docentes de primaria en España. Al mismo tiempo, hemos de tener en consideración que las universidades disponen de potestad para la asignación que consideren de 30 créditos más en cualquiera de los módulos que plantea la citada Orden, por lo que el cálculo es aproximado.
} 
Dentro de este apartado, podemos comprobar, tanto en España como en Argentina, que la formación profesional específica tiene un peso muy elevado del total de la carga lectiva de los estudios del profesorado de educación infantil (87,5\% y 75\% respectivamente).

Por el contrario, Colombia presenta una formación de 2,5 años (2 años si provienen de un bachiller pedagógico) en el itinerario de las Escuelas Normales superiores que sería el más frecuentado en el caso de los profesores de Primaria, mientras que en el caso francés la formación estrictamente de formación del profesorado es de dos años aun cuando la totalidad de la formación implica 5-6 años.

Es interesante constatar como la duración de la formación no implica que la entrada como docentes en el sistema se realice a la misma edad. Si analizamos el caso de un alumnos/a estándar, En Argentina y España podrán entrar como docentes en el sistema educativo a partir de los 22 años (aun cuando la situación laboral española hará que se retrase bastante esa edad), los colombianos pueden hacerlo a partir de los 18-19 años, mientras que los franceses lo harán después de los 25 años.

\subsubsection{Iniciación al desarrollo profesional docente}

En ninguno de los países estudiados existen programas de inducción. Sin embargo, se establecen medidas de apoyo en todos ellos (de carácter autonómico en el caso de España, mientras que en el resto son de carácter estatal). Si bien, como ya advertimos en la nota 7 de la tabla comparativa respecto de Argentina, la línea de acción destinada al acompañamiento de maestros y profesores en sus primeros desempeños profesionales, como práctica regular y sistemática derivada de la acción estatal no es generalizada, sino que se desarrolla exclusivamente en los Institutos de Formación Superior seleccionados por las provincias. En España, tal y como explica Comisión Europea/EACEA/Eurydice (2013) las Comunidades Autónomas establecen las medidas de apoyo que se ofrecerán a los docentes durante el período de prueba (fase de prácticas) al punto que se garantiza la oferta de todo tipo de medidas de apoyo para los docentes de nueva incorporación, tales como: reuniones periódicas para debatir sobre avances o problemas; asistencia en la planificación y evaluación de las clases; tutorización; participación en las clases de otros profesores u observación en el aula; formación especial obligatoria y visitas a otras escuelas/centros de recursos.

En lo que respecta al método de acceso a la profesión docente, podemos apreciar que, en España (en un centro público de enseñanza) es el concurso-oposición, mientras que en Argentina y Colombia es sólo mediante concurso. Eso sí, en todos los casos tras finalizar la formación inicial docente. Francia también establece una fase de concurso, con la salvedad de que los futuros docentes todavía están cursando el período de formación inicial. Vamos a matizar sucintamente cada una de las situaciones que presentan los países.

Así, en el caso de España, para la enseñanza pública:

la prueba consta de tres fases: una fase de examen en la que se evalúan los conocimientos específicos en el campo o la especialidad correspondiente, la aptitud pedagógica y el dominio de las técnicas didácticas necesarias; una fase de selección en base a los méritos, en la que se evalúan, en función de lo establecido en cada convocatoria, la idoneidad de los candidatos (formación académica y la experiencia docente previa ); y un período de prueba durante el cual los candidatos seleccionados han de demostrar su aptitud para la enseñanza (Comisión Europea/EACEA/Eurydice, 2013, pp. 48-49).

En lo que respecta a la enseñanza privada y concertada, se trata de un contrato después de las correspondientes entrevistas de selección. Además, en el caso de la concertada, ese contrato ha de contar con la aprobación del Consejo Escolar del centro. 
Por su parte, Argentina, tal y como hemos explicado en la Tabla 1, el Estatuto docente establece que el ingreso al ejercicio en la enseñanza primaria será por concurso. No obstante, se establece el complemento de pruebas de oposición en los casos en que se consideren necesarias. Entre los antecedentes que la Junta de Clasificación y disciplina debe considerar se encuentra: los títulos docentes, el promedio de calificaciones, la antigüedad del título o los títulos exigibles, así como la antigüedad de gestiones (art.63). Y para algunas modalidades (maestro de escuelas para adultos, carcelarias y hospitalarias) se establecen 5 años de antigüedad en el ejercicio de la docencia en las escuelas comunes. Si bien es cierto que si no hay aspirantes que reúnan estas condiciones podrían ser designados con menor antigüedad e incluso sin ella (art 65) (MED, 1958).

En el caso colombiano, el docente titulado debe presentarse a un concurso cuyo resultado le dará un puntaje que le permitirá elegir un destino, inicialmente cada año y posteriormente cada período de tres-cinco años. El puntaje irá incrementándose por cursos realizados, publicaciones, etc.

Por último, en Francia existe un concours al final del Master 1 en la ESPE para acceder al Master 2. Los alumnos que acceden son considerados funcionarios en formación y en consecuencia reciben un salario. Una vez terminada su formación, son destinados por l'Académie (semejante en muchos aspectos a una Consejería de Educación autonómica) correspondiente a su escuela primaria de destino.

\section{Conclusiones}

Los cuatro países analizados están en la órbita de los sistemas comprensivos y de la formación inicial de los docentes de tendencia simultánea. La excepción es Francia, tanto en cuanto a la diferenciación de su sistema en los últimos años de escolaridad obligatoria como en su sistema consecutivo de formación de maestros de primaria, único en el contexto europeo.

Esta circunstancia no puede ser analizada de forma separada de los años y nivel de formación y de la edad habitual de los docentes en el final de su formación. Si observamos el cuadro de yuxtaposición, nos percatamos que puede haber una diferencia de hasta 8 años de edad entre el docente inicial francés y el colombiano, lo que indica claramente una sustancial diferencia tanto a nivel de cantidad de formación recibida como a nivel de madurez personal.

Sin duda la personalidad del docente que llega a su primera clase está influida por esta situación, conocimientos y madurez personal, pero al mismo tiempo también lo está por la preparación pedagógica y la práctica docente $\mathrm{y}$, en ese sentido, los docentes españoles, argentinos o incluso colombianos, que provienen de un sistema de formación simultáneo llegan con mayor bagaje pedagógico a la escuela.

Esto si aceptamos que la formación inicial de los docentes es un camino que conduce a la práctica profesional como enseñante. Obvio diríamos. Pero solo es obvio en el sistema francés que al tener la selección a la entrada del último año de formación y los estudiantes ser pagados como "docentes en formación" hace que casi el $100 \%$ de los mismos acabe en la escuela. Pero esto no ocurre así en los demás países. En España solo el $20 \%$ de los titulados obtiene un trabajo como docente en los tres años posteriores a finalizar sus estudios, mientras que, en Colombia, un alto porcentaje utiliza su título para acceder a la universidad en alguna carrera de carácter pedagógico y en Argentina más de la mitad de los profesores que inician su trabajo lo comparten con estudios universitarios en carreras educativas. 
Uno de los aspectos que están pendientes de análisis es la incidencia fuerte que en el caso español ha tenido el cambio de perfil de alumnado como consecuencia del establecimiento del cupo único de entrada a la formación sin establecer porcentajes como hasta hace unos años para aquellos que provenían del bachillerato o de la formación Profesional. Esta circunstancia ha supuesto un cambio de perfil que se nota de manera más intensa en los alumnos/as que acceden al Magisterio de Educación Infantil, como se observa en la tabla anterior. Esta circunstancia, muy específica de España, no ha sido aún evaluada en sus diferentes aspectos, entre ellos los correspondientes a su incidencia en los niveles de calidad de la formación.

Uno de los elementos que se muestra relevante en una formación docente de calidad ${ }^{2}$ es el componente de formación en investigación educativa que, normalmente, suele visibilizarse y culmina con un trabajo de investigación durante el período de formación. En este caso, es destacable el hecho de que sólo en España y Francia se lleve a cabo este tipo de formación, lo que nos permite atisbar en los planes de estudio de estos países una concepción más amplia del perfil del futuro docente, no sólo centrada en la profesionalización del ejercicio docente en las aulas, sino también en la integración de éste en procesos de investigación conscientes que permitan aplicar sus resultados en el aula en un claro proceso de mejora continua de la docencia.

Respecto al apoyo que reciben de la administración en su iniciación como docentes, las informaciones no son de demasiada utilidad, pues con frecuencia la concreción de lo establecido en las normativas tiene una interpretación muy diversa. En el caso español, por ejemplo, el primer año de trabajo se considera un período de prueba donde el docente recibe ayuda por parte del centro y de la propia administración educativa a través de diversas vías, desde el centro, desde los centros de profesores, desde las propias Consejerías de educación, etc.- cuando en la práctica, en la mayoría de las Comunidades Autónomas solo recibe un cierto apoyo desde el centro si hay buena voluntad en el equipo directivo. Algo similar ocurre en Colombia y Argentina, donde la soledad del docente, especialmente si trabaja en el medio rural, es muy grande y es causa de muchas deserciones en el trabajo.

Como se observa, la interpretación del análisis comparativo de la formación inicial docente es compleja y sólo puede ser entendida si se realiza desde la combinación de los análisis de variables, pues éstas son interdependientes y modifican los resultados de una interpretación unívoca de cada una de ellas. Esto resulta especialmente importante si deseamos obtener resultados respecto a la calidad de los docentes, que no parece ligada solamente al sistema de formación inicial sino casi al conjunto de variables analizadas en nuestro trabajo.

\section{Referencias bibliográficas}

Bereday, G. F. (1964). El método comparativo en pedagogía. Barcelona: Herder.

CFE (2007b). Resolución CFE N.24/07, de 7 de noviembre de 2007. Lineamientos Curriculares Nacionales para la Formación Docente Inicial. Recuperado de

2

"La adquisición de conocimientos y competencias sobre investigación educativa se ha señalado como un elemento importante de la formación del profesorado, que debe ayudar a los profesores a incorporar los resultados tanto de la investigación académica como de la investigación en el aula a su docencia" (Comisión Europea/EACEA/Eurydice, 2013, p.30). 
http://repositorio.educacion.gov.ar/dspace/bitstream/handle/123456789/89847/linea mientos_curriculares $\% 5 \mathrm{~b} 1 \% 5 \mathrm{~d}$.pdf?sequence $=1$

CFE (2007a). Resolución CFE N.30/07, de 29 de noviembre. Anexo I: Hacia una Institucionalidad del Sistema de Formación Docente en Argentina. Recuperado de http://repositorio.educacion.gov.ar:8080/dspace/bitstream/handle/123456789/53996/ 12930.pdf? sequence $=1$

COMISIÓN EUROPEA/EACEA/EURYDICE (2013). Cifras clave del profesorado y la dirección de centros educativos en Europa. Edición 2013. Informe de Eurydice. Luxemburgo: Oficina de Publicaciones de la Unión Europea.

Ferrer Julià, f. (1990). Educación Comparada. Fundamentos teóricos, metodología y modelos. Barcelona: PPU.

García Garrido, J.L. (1982). Educación Comparada. Fundamentos y problemas. Madrid: Dykinson.

García Garrido, J.L. y García Ruiz, M.J. (2012). La metodología de la Educación Comparada. En J.L. García Garrido, M.J. García Ruiz, y E. Gavarie Starkie. La Educación Comparada en tiempos de globalización. Madrid: UNED.

Ginestie, J (2014). Système éducatif et formation des enseignants en France. Recuperado de https://www.academia.edu/987726/SYST\%C3\%88ME_\%C3\%89DUCATIF_ET_FO RMATION_DES_ENSEIGNANTS_EN_FRANCE

Hilker, F (1968): La Pédagogie Comparée. París: Institute Pédagogic National.

MED (1958). Ley 14.473 de 12 de septiembre de 1958, Estatuto del docente. Boletín Oficial, de 12 de septiembre de 1958.

MECD (2007a). Real Decreto 1393/2007, de 29 de octubre, por el que se establece la ordenación de las enseñanzas universitarias oficiales. Boletín Oficial del Estado, $\mathrm{n}^{\circ}$ 260, de 30 de octubre de 2007.

MECD (2007b). ORDEN ECI/3857/2007, de 27 de diciembre, por la que se establecen los requisitos para la verificación de los títulos universitarios oficiales que habiliten para el ejercicio de la profesión de Maestro en Educación Primaria. Boletín Oficial del Estado, $\mathrm{n}^{\circ} 312$, de 29 de diciembre de 2007.

MECD (2013). Ley Orgánica 8/2013, de 9 de diciembre, para la mejora de la calidad educativa (LOMCE). Boletín Oficial del Estado, nº 295, 2013, de 10 diciembre de 2013.

MECD (2014). Real Decreto 412/2014, de 6 de junio, por el que se establece la normativa básica de los procedimientos de admisión a las enseñanzas universitarias oficiales de Grado. Boletín Oficial del Estado, no 138, de 7 de junio de 2014.

MEN-Ministerio de Educación Nacional (2012). Políticas y sistema colombiano de formación y Desarrollo profesional docente. Recuperado de http://www.mineducacion.gov.co/cvn/1665/articles-208603_archivo_pdf.pdf 
MEN-Ministère d'Éducation Nationale (2016). Formation des enseignants: éléments de comparaison internationale. Recuperado de http://cms.unige.ch/asso-etud/adefep/wpcontent/uploads/2014/04/consulter_la_comparasion_internationale_sur_la_formation _des_enseignants2.pdf

OEI (2003). Red Quipu. Estructura y organización de la formación docente en Iberoamérica. Recuperado de http://www.oei.es/quipu/argentina/index.html

OEI (2015). Red Quipu. Información sistemas educativos iberoamericanos. Recuperado de http://www.oei.org.co/oeimad/quipu.htm

OREALC/UNESCO (marzo 2007). Educación de calidad para todos: Un asunto de Derechos Humanos. Documento de discusión sobre políticas educativas en el marco de la II Reunión Intergubernamental del Proyecto Regional de Educación para América Latina y El Caribe (EPT/PRELAC). Buenos Aires: OREALC/UNESCO.

Reis, P. (2016, abril). Formación de profesores. Perspectivas de Brasil, Colombia, España y Portugal. Revista electrónica Interuniversitaria de Formación del Profesorado [en línea], $\quad \mathrm{N}^{\circ} 54 . \quad$ Recuperado de http://aufop.com/aufop/uploaded_files/revistas/146246747510.pdf $\quad[2016,15$ de junio].

Roselló, P. (1978). Teoría de las corrientes educativas. Barcelona: Promoción Cultural.

Schriewer, J (1993). El método comparativo y la necesidad de externalización: criterios metodológicos y conceptos sociológicos. En J. Schriewer y F. Pedró (eds.). Manual de Educación Comparada. Vol.2. Teorías, investigaciones y perspectivas. Barcelona: PPU.

UNESCO/IBE (2010). Datos mundiales de la educación 2010/2011. VII Edición. Paris: UNESCO/IBE. 
